\title{
EFECTO DEL ACONDICIONADO PREVIO AL ALMACENAJE REFRIGERADO SOBRE LA CALIDAD DE CIRUELAS ‘CONSTANZA’ $\left({ }^{1}\right)$
}

\author{
EDUARDO SEIBERT $\left(2^{*}\right)$; SUSANA GONZÁLEZ $(3)$; ARIEL ORELLANA $\left({ }^{3}\right)$; \\ LUIS LUCHSINGER $\left({ }^{4}\right)$; RENAR JOÃO BENDER $\left({ }^{5}\right)$
}

\begin{abstract}
RESUMEN
El efecto del acondicionado a $20^{\circ} \mathrm{C}$ por 6 días previo al almacenaje refrigerado sobre la calidad y el desarrollo de daños por frío fue estudiado en ciruelas 'Constanza'. Terminado el tratamiento las ciruelas fueron almacenadas a $0{ }^{\circ} \mathrm{C}$ por $7,14,21,28,35$ y 42 días, o a $5{ }^{\circ} \mathrm{C}$ por 7,14 y 21 días, con evaluaciones a la cosecha, en cada salida de frío y después de madurar a $20^{\circ} \mathrm{C}$. Como control las frutas fueron almacenadas a 0 y $5{ }^{\circ} \mathrm{C}$, sin acondicionar. Las pérdidas de masa fresca fueron bajas durante el almacenaje a 0 o $5^{\circ} \mathrm{C}$ y aumentaron en la maduración. La firmeza cambió poco en las ciruelas-control a $0{ }^{\circ} \mathrm{C}$, mientras que en las acondicionadas disminuyó hasta los 28 días a $0{ }^{\circ} \mathrm{C}$. El acondicionado combinado con el almacenaje a 5 ${ }^{\circ} \mathrm{C}$ causó una gran pérdida de firmeza, con un $50 \%$ de frutos presentando firmeza baja para comercialización en la maduración. El contenido de jugo fue superior en los frutos acondicionados y almacenados a $0{ }^{\circ} \mathrm{C}$ respecto a los controles, pero no existieron diferencias entre los tratamientos en las ciruelas almacenadas a $5{ }^{\circ} \mathrm{C}$. Harinosidad se visualizó en frutos acondicionados y controles a 0 o $5{ }^{\circ} \mathrm{C}$, sin diferencias entre los tratamientos. Los frutos controles, en la maduración, presentaron pardeamiento pasados 42 días a $0{ }^{\circ} \mathrm{C}$ y retención de firmeza pasados 28, 35 y 42 días a $0{ }^{\circ} \mathrm{C}$. El acondicionado causó una tendencia de aumento del porcentaje de frutos sanos en la maduración pasados 28,35 y 42 días a $0{ }^{\circ} \mathrm{C}$.
\end{abstract}

Palabras clave: Frutos de carozo, ciruela, calentamiento, refrigeración, daños por frío.

\section{ABSTRACT \\ DELAYED COOLING EFFECT ON THE QUALITY OF ‘CONSTANZA’ PLUMS}

The effects of delayed cooling (DC) at $20^{\circ} \mathrm{C}$ for 6 days before cold storage was studied in 'Constanza' plums evaluating development of quality and chilling injuries. At the end of the DC treatment the plums were cold stored at $0{ }^{\circ} \mathrm{C}$ for $7,14,21,28,35$ or 42 days or at $5{ }^{\circ} \mathrm{C}$ for 7,14 or 21 days and evaluated at harvest, after cold storage periods and after a ripening period at $20{ }^{\circ} \mathrm{C}$. The control fruits were stored at 0 or $5{ }^{\circ} \mathrm{C}$. Weight loss was low during storage at 0 or $5{ }^{\circ} \mathrm{C}$ and increased during ripening periods. Firmness changed little in control plums at $0{ }^{\circ} \mathrm{C}$ while in DC plums decreased constantly up to 28 days. Delayed cooling treatment resulted in significant decreases of firmness of plums stored at $5{ }^{\circ} \mathrm{C}$, which presented $50 \%$ of fruit with unacceptable firmness for commercialization after the ripening periods starting from 7 days of storage. Extractable juice was higher in $\mathrm{DC}$ fruit than in control fruits at $0^{\circ} \mathrm{C}$, but there were no differences in juiceness in plums stored at $5{ }^{\circ} \mathrm{C}$. Woolliness was visualized at ripening in DC and control fruits stored at 0 or $5^{\circ} \mathrm{C}$ without differences among treatments. At ripening, control fruit presented internal browning after 42 days at $0{ }^{\circ} \mathrm{C}$ and leatheriness after 28,35 and 42 days at $0{ }^{\circ} \mathrm{C}$. Delayed cooling induced an increase of the percentage of healthy fruit after 28,35 and 42 days at $0{ }^{\circ} \mathrm{C}$.

Key words: Stone fruits, plum, conditioning, cold storage, chilling injury.

( $\left.{ }^{1}\right)$ Recibido para publicación el 17 de mayo de 2006 y aceptado el 20 de agosto de 2007.

$\left({ }^{2}\right)$ Escola Agrotécnica Federal de Sombrio, Santa Rosa do Sul, SC. E-mail: eduseibert@ig.com.br $\left(^{*}\right)$ Autor correspondente.

$\left({ }^{3}\right)$ Nucleo Milenio en Biología Celular Vegetal, Centro de Biotecnología Vegetal, Universidad Andrés Bello, Chile. E-mail: ushi_su77@yahoo.com.ar; aorellana@unab.cl

$\left({ }^{4}\right)$ Centro de Estudios Postcosecha, Facultad de Ciencias Agronómicas, Universidad de Chile, Santiago, Chile. E-mail: 1luchsin@uchile.cl

$\left({ }^{5}\right)$ Laboratório de Fisiologia Pós-colheita, Faculdade de Agronomia, UFRGS, Porto Alegre, RS. E-mail: rjbe@vortex.ufrgs.br 


\title{
RESUMO \\ EFEITO DO ATRASO NA ARMAZENAGEM REFRIGERADA SOBRE A QUALIDADE DE AMEIXAS ‘CONSTANZA'
}

\begin{abstract}
Estudou-se o efeito do condicionamento $(\mathrm{CD})$ a $20^{\circ} \mathrm{C}$ por seis dias antes da armazenagem refrigerada sobre a qualidade e o desenvolvimento de danos por frio em ameixas 'Constanza'. Terminado o tratamento, as ameixas foram armazenadas a $0{ }^{\circ} \mathrm{C}$ por $7,14,21,28,35$ e 42 dias ou a $5{ }^{\circ} \mathrm{C}$ por 7,14 e 21 dias, e avaliadas na colheita, em cada saída da armazenagem refrigerada e após amadurecidas a $20^{\circ} \mathrm{C}$. Como controle, as frutas foram armazenadas a 0 e $5{ }^{\circ} \mathrm{C}$, sem condicionar. As perdas de massa fresca foram pequenas durante a armazenagem a 0 ou $5{ }^{\circ} \mathrm{C}$ e aumentaram no amadurecimento. A firmeza pouco variou nas ameixas-controle durante a armazenagem a $0{ }^{\circ} \mathrm{C}$, enquanto nas condicionadas diminuiu até os 28 dias. A combinação do $\mathrm{CD}$ com a armazenagem a $5{ }^{\circ} \mathrm{C}$ levou à grande perda de firmeza, observando-se em $50 \%$ dos frutos baixa firmeza para comercialização no amadurecimento. O conteúdo de suco foi maior nos frutos condicionados e armazenados a $0^{\circ} \mathrm{C}$ quando comparado aos controles, mas não houve diferenças entre as ameixas armazenadas a $5{ }^{\circ} \mathrm{C}$. Foi visualizada lanosidade no amadurecimento em frutos-controle e condicionados, sem diferenças entre os tratamentos, a 0 ou $5^{\circ} \mathrm{C}$. No amadurecimento, nos frutos-controle houve escurecimento após 42 dias a $0{ }^{\circ} \mathrm{C}$ e retenção de firmeza após 28,35 e 42 dias a $0{ }^{\circ} \mathrm{C}$. $\mathrm{O}$ condicionamento induziu aumento na porcentagem de frutos sadios no amadurecimento após 28,35 e 42 dias a $0^{\circ} \mathrm{C}$.
\end{abstract}

Palavras-chave: Frutas de caroço, ameixa, condicionamento, refrigeração, danos por frio.

\section{INTRODUCCIÓN}

La producción mundial de ciruelas en 2005 fue de aproximadamente 9,8 millones de toneladas siendo China, Estados Unidos de America y Yugoslávia los principales países productores. En América del Sur, sólo Chile con 255 mil toneladas y Argentina con 127 mil toneladas producidas en 2005, figuran como grandes productores. Además de ser un gran productor, Chile es un de los principales países exportadores de ciruelas, exportando aproximadamente 103 mil toneladas en 2004 (FAO, 2006). Sin embargo, las exigencias de calidad son cada año superiores en los mercados de exportación de frutas de carozo, lo que ha sido causal del estancamiento de los volúmenes exportados y precios en las últimas temporadas (LuCHSINGER Y WALSh, 1997).

La refrigeración es la principal técnica usada para preservar la calidad de frutas y otros productos perecibles. Una vez que la fruta es cosechada debe ser almacenada y transportada a bajas temperaturas para extender su vida de postcosecha y así, llegar a los mercados consumidores. Pero esto muchas veces no es suficiente para retardar los cambios que afectan la calidad. Las bajas temperaturas pueden conducir al aparecimiento de daños por frío. Frutos de carozo presentan daños por frío cuando son expuestos durante largos períodos a temperaturas bajo $\operatorname{los} 8^{\circ} \mathrm{C}$. Estos daños causan desórdenes fisiológicos que en ciruelas se pueden presentar como pardeamiento interno, transparencia de la pulpa y harinosidad, limitando la vida postcosecha y disminuyendo la calidad de los frutos (CRIsosto el al., 1999). Así como en otros frutos, en ciruelas estos daños presentan intensidad variable entre las variedades y no son visualizados externamente, sino cuando los frutos son cortados en el momento del consumo.

La presencia de desórdenes fisiológicos compromete un importante porcentaje de la fruta almacenada, y es uno de los problemas detectados en los mercados consumidores de ciruelas (Lizana y Rodriguez, 1995). Según BruHn (1991), para los consumidores de Califórnia los mayores problemas de frutas de carozo son la falta de aroma y sabor, la textura harinosa y el hecho que no alcanzan maduración para consumo. De estos consumidores, $50 \%$ pagarían más por frutas sanas, maduras y con buen sabor y olor. De ahí la importancia de estudiar las condiciones de almacenaje para que la fruta tenga mejor calidad y duración en postcosecha y la venta en los países compradores supere los 28 días (LizANA y RoDRIGUEZ, 1995).

Algunas estrategias para prevenir el aparecimiento de daños por frío han sido investigadas. Algunas son el control de la atmósfera durante el almacenaje, el uso de variedades menos susceptibles, el uso del calentamiento intermitente y del atraso en el almacenaje refrigerado conocido como acondicionado o calentamiento.

El acondicionado a $20{ }^{\circ} \mathrm{C}$ antes del almacenaje refrigerado, para aumentar la maduración de los frutos, está siendo muy usado para disminuir los síntomas de daños por frío. Sin embargo, los resultados dependen de la variedad. La literatura es ambigua incluyendo relatos tanto de reducción en los daños, como de ausencia de resultados. Los trabajos 
también mencionan alta pérdida de masa y firmeza de los frutos (Retamales et al., 1992; STReif et al., 1994; Zhou et al., 2000; CRISOsto el al., 2004).

El objetivo del trabajo fue evaluar el comportamiento y la calidad postcosecha de ciruelas 'Constanza' expuestas al acondicionado a $20{ }^{\circ} \mathrm{C}$ previo el almacenamiento refrigerado a 0 y $5^{\circ} \mathrm{C}$.

\section{MATERIAL Y MÉTODOS}

El ensayo se realizó con frutos de ciruelo japonés variedad Constanza, de cosecha tardía correspondiente a mediados de abril. Los frutos con 35,2 N de firmeza de pulpa fueron cosechados en un huerto ubicado en la Región Metropolitana, Chile. Se realizó una cosecha y la fruta fue transportada de inmediato al Centro de Estudios Postcosecha (CEPOC) de la Facultad de Ciencias Agronómicas de la Universidad de Chile, donde los frutos fueron seleccionados y embalados de acuerdo con las normas establecidas para exportación en cajas de cartón de $8,2 \mathrm{~kg}$.

Los frutos embalados fueron separados en dos grupos y almacenados a 0 y $5{ }^{\circ} \mathrm{C}$, con un $90 \%$ de humedad relativa por $7,14,21,28,35$ y 42 días para los frutos a $0{ }^{\circ} \mathrm{C}$ y por $7,14,21$ días para los frutos a $5{ }^{\circ} \mathrm{C}$, formando los tratamientos-control. El otro grupo de frutos fue sometido al acondicionado, que consistió en almacenar los frutos en una cámara a $20^{\circ} \mathrm{C}$ por 6 días para aumentar su maduración. Terminado el acondicionado los frutos fueron almacenados a 0 y 5 ${ }^{\circ} \mathrm{C}$, por los mismos períodos de tiempo que los frutoscontrol. Los frutos se analizaron a la cosecha y en cada salida de frío seguidos de un período de maduración a $20^{\circ} \mathrm{C}$, variable para cada tratamiento.

La deshidratación fue evaluada por pérdida de masa a salida de cada período de almacenamiento. El color de cubrimiento y de la pulpa se evaluó con un colorímetro Minolta CR300, escala CIELab, fuente de iluminante $\mathrm{D}_{65} \mathrm{y}$ ángulo de observador de $2^{\circ}$, expresando los resultados como luminosidad $\left(\mathrm{L}^{*}\right)$, pureza $\left(\mathrm{C}^{*}\right)$ y tonalidad $\left(\mathrm{H}_{\mathrm{ab}}\right)$. La firmeza de la pulpa, expresa en Newtons $(\mathrm{N})$, fue medida en la zona ecuatorial (ambas caras), sutura, hombro y punta, con un presionómetro manual con embolo de $7,9 \mathrm{~mm}$ de diámetro, tras remover la piel en la zona de medición. El contenido de jugo fue medido subjetiva y objetivamente. En la evaluación subjetiva visual se partieron los frutos por la zona ecuatorial en dos mitades y apretándose una mitad con la mano se clasificó el grado de jugosidad en: 1 = Alta (abundante liberación de jugo), 2 = Moderada (moderada liberación de jugo), 3 = Baja (leve liberación de jugo), 4 = Nula (sin jugo). En la medición objetiva fue usado el método de LILL y VAN Der Mespel (1988) modificado por Luchsinger (2000). Trozos de pulpa sacados de la zona ecuatorial de los frutos, fueron homogeneizados a través de una jeringa sin aguja de $5 \mathrm{~mL}$, colectados en tubos de centrifugación y centrifugados a $12.000 \mathrm{gn}$ por 20 minutos siendo el jugo sobrenadante colectado y pesado. El contenido de jugo fue calculado por: $X$ $=[($ masa del sobrenadante $/$ masa de pulpa $) \times 100]$. Los sólidos solubles totales (SST) fueron medidos con un refractómetro Atago en una muestra de jugo de cada fruto y expresados como ${ }^{\circ} \mathrm{Brix}$.

El grado de pardeamiento interno se evaluó visualmente, separándose los frutos por la zona ecuatorial en dos mitades para observar la coloración del tejido y clasificar por una escala, donde 1 = Sano (pulpa se presenta normal), 2 = Leve $(0 \%-25 \%$ de la pulpa parda), $3=$ Moderado $(25 \%-$ $50 \%$ de la pulpa parda), $4=$ Severo $(>50 \%$ de la pulpa parda). La harinosidad fue evaluada subjetiva y objetivamente. En la forma subjetiva visual del grado de harinosidad, se partieron los frutos por la zona ecuatorial en dos mitades y apretando-se una mitad con la mano se clasificó por una escala, donde 1 = Sano (abundante liberación de jugo), 2 = Leve (moderada liberación de jugo), 3 = Moderado (leve liberación de jugo), $4=$ Severo (sin jugo). Frutos secos, con poco o sin jugo fueron clasificados como harinosos.

En la forma objetiva se uso el método de LUCHSINGER (2000). Se elaboró una curva que relaciona la firmeza de la pulpa con el contenido de jugo en los 6 días siguientes a la cosecha a $20{ }^{\circ} \mathrm{C}$ para la evaluación que expresa el contenido de jugo disponible en el fruto al simular una maduración normal sin almacenaje refrigerado. Después, en cada fecha de evaluación, se comparo el contenido de jugo y la firmeza de cada fruto con la curva "jugo vs. firmeza" y el índice de harinosidad se expreso como: $X=100-\left[100 \times\right.$ (contenido de jugo a 0,5 o $\left.20^{\circ} \mathrm{C}\right) /$ (contenido de jugo a $20^{\circ} \mathrm{C}$, a la cosecha)], en que $\mathrm{X}=$ 0 representa un fruto con contenido normal de jugo y $X=100$ un fruto totalmente seco. Frutos jugosos y sin daños por frío fueron considerados sanos y aptos para su comercialización.

El experimento presentó un diseño completamente aleatorizado, con 20 repeticiones por tratamiento y fecha de evaluación, siendo el fruto la unidad experimental. El análisis de varianza se realizó por el sistema de análisis estadístico SANEST (Zonta y Machado, 1986) empleando el test de Tukey al $5 \%$. 


\section{RESULTADOS Y DISCUSIÓN}

No se observaron pudriciones en los frutos durante todo el período del ensayo. Además de la ausencia de pudriciones, las ciruelas presentaron buena coloración y aspecto hasta el final del almacenaje y período de maduración.

La deshidratación por pérdida de masa fue pequeña a la salida de la conservación para las dos temperaturas de almacenaje, siendo que sólo después de 35 días a $0{ }^{\circ} \mathrm{C}$ supero los $2 \%$ (Tabla 1) cuando los frutos acondicionados pasaron a presentar mayor deshidratación. KLuge et al. (1997) observaron pérdidas de masa en ciruelas 'Santa Rosa' de 0,9 a $1,3 \%$ durante 24 días a $0{ }^{\circ} \mathrm{C}$ y de 5,9 a $6,3 \%$ en más 3 días adicionales de maduración a $20^{\circ} \mathrm{C}$. A salida de maduración hubo alzas en la pérdida de masa en relación a la salida de frío, debido a la alta temperatura y baja humedad en este ambiente $\left(20^{\circ} \mathrm{C}\right.$ y $\left.60-70 \% \mathrm{HR}\right)$. Hasta los 14 días a $0{ }^{\circ} \mathrm{C}$ no existieron diferencias significativas, pero las ciruelas acondicionadas presentaron menor pérdida de masa a partir de los 21 días. Una menor pérdida de masa también ocurrió en las ciruelas acondicionadas y almacenadas a $5^{\circ} \mathrm{C}$. Sin embargo, esta deshidratación en los frutos acondicionados después de 21 días a $0{ }^{\circ} \mathrm{C}$ y 7 días a $5{ }^{\circ} \mathrm{C}$ no explica todo lo ocurrido, pues estos frutos se quedaron por menos tiempo a $20^{\circ} \mathrm{C}$. Eso ocurrió debido a su baja firmeza lo que llevo a su evaluación con menos días en el período de maduración a $20^{\circ} \mathrm{C}$. Si hubiesen quedado en maduración el mismo tiempo que las ciruelas-control, posiblemente tendrían una pérdida de masa superior como observaron RetAMALES et al. (1992) y STREIF et al. (1994). Según estos autores el acondicionado causa una gran pérdida de masa en los frutos.

Tabla 1. Porcentaje de deshidratación en ciruelas 'Constanza' a la salida del almacenaje refrigerado y después de maduración a $20^{\circ} \mathrm{C}$

\begin{tabular}{|c|c|c|c|c|c|c|c|c|}
\hline \multirow{3}{*}{$\begin{array}{l}\text { Fechas } \\
\text { de análisis }\end{array}$} & \multicolumn{4}{|c|}{ Almacenamiento a $0{ }^{\circ} \mathrm{C}$} & \multicolumn{4}{|c|}{ Almacenamiento a $5{ }^{\circ} \mathrm{C}$} \\
\hline & \multicolumn{2}{|c|}{ Almacenaje } & \multicolumn{2}{|c|}{ Maduración } & \multicolumn{2}{|c|}{ Almacenaje } & \multicolumn{2}{|c|}{ Maduración } \\
\hline & Control & $\mathrm{AC}$ & Control & $\mathrm{AC}$ & Control & $\mathrm{AC}$ & Control & $\mathrm{AC}$ \\
\hline 0 & -- & -- & $1,7 \mathrm{a}$ & $2,1 \mathrm{a}$ & -- & -- & $1,3 \mathrm{~b}$ & $1,7 \mathrm{a}$ \\
\hline 7 & $0,0 \mathrm{a}$ & $0,0 \mathrm{a}$ & 4,6 a & $4,7 \mathrm{a}$ & $0,0 \mathrm{a}$ & $0,0 \mathrm{a}$ & 5,0 a & $2,3 \mathrm{~b}$ \\
\hline 14 & $0,2 \mathrm{a}$ & $0,3 \mathrm{a}$ & $5,5 \mathrm{a}$ & 5,6 a & $0,8 \mathrm{a}$ & $0,2 \mathrm{a}$ & $4,7 \mathrm{a}$ & $3,5 \mathrm{~b}$ \\
\hline 21 & $0,8 \mathrm{a}$ & $0,6 \mathrm{~b}$ & 5,6 a & $4,5 \mathrm{~b}$ & $1,9 \mathrm{a}$ & 1,6 a & 3,4 a & $2,7 \mathrm{~b}$ \\
\hline 28 & $1,8 \mathrm{a}$ & $1,2 \mathrm{~b}$ & $5,9 \mathrm{a}$ & $3,4 \mathrm{~b}$ & -- & -- & -- & -- \\
\hline 35 & $2,2 \mathrm{~b}$ & $2,8 \mathrm{a}$ & $7,2 \mathrm{a}$ & $4,0 \mathrm{~b}$ & -- & -- & -- & -- \\
\hline 42 & $3,2 \mathrm{~b}$ & 4,4 a & $6,5 \mathrm{a}$ & $4,4 \mathrm{~b}$ & -- & -- & -- & -- \\
\hline Promedio & $1,4 \mathrm{a}$ & $1,5 \mathrm{a}$ & $5,3 \mathrm{a}$ & $4,1 \mathrm{~b}$ & $0,9 \mathrm{a}$ & $0,8 \mathrm{a}$ & $3,6 \mathrm{a}$ & $2,6 \mathrm{~b}$ \\
\hline $\mathrm{CV}(\%)$ & \multicolumn{2}{|c|}{9,3} & \multicolumn{2}{|c|}{15,9} & \multicolumn{2}{|c|}{19,9} & \multicolumn{2}{|c|}{7,2} \\
\hline
\end{tabular}

- Indica que la variable no presenta evaluación en esta fecha. Valores seguidos de la misma letra en la línea, dentro de cada temperatura de almacenaje y período de evaluación, no difieren significativamente por el test de Tukey $(p=0,05)$. AC $=$ Acondicionado. CV $=$ Coeficiente de variación.

Los frutos presentaron visualmente el 100\% de la piel con coloración morado oscuro para morado. No se presentaron diferencias de luminosidad $\left(\mathrm{L}^{*}\right)$ entre los tratamientos durante la conservación a 0 o $5{ }^{\circ} \mathrm{C}$ y durante la maduración (Tabla 2). Se detectaron disminuciones para $\mathrm{C}^{*}$, con valores menores en las ciruelas acondicionadas, indicando un color rojo más apagado (menor saturación). Los valores del ángulo $\mathrm{H}_{\mathrm{ab}}$ oscilaron en ambos tratamientos y temperaturas de almacenaje, con diferencias sólo en la maduración en frutos almacenados a $0{ }^{\circ} \mathrm{C}$, mientras que los acondicionados presentaron levemente mayor ángulo $\mathrm{H}_{\mathrm{ab}}$ indicando que tenían una mayor tonalidad roja (Tabla 2).
La pulpa cambió de la coloración amarilla o naranja en los frutos almacenados en frío, para roja o morado en los maduros. La luminosidad promedio en la pulpa fue menor en los frutos acondicionados durante la conservación y maduración (Tabla 2). La pureza $\left(\mathrm{C}^{*}\right)$ del color disminuyó en el almacenaje y maduración, siendo el descenso mayor en los frutos acondicionados, que presentaron un menor valor de $\mathrm{C}^{*}$. El ángulo $\mathrm{H}_{\mathrm{ab}}$ fue menor en los frutos acondicionados, que en los controles disminuyendo durante el almacenaje a $0^{\circ} \mathrm{C}$ y su maduración, indicando que presentaban una coloración más rojiza debido al aumento de madurez (Figura 1A y 1B), cambiando de una tonalidad naranja para una rojiza 
intensa pasados 30 días en frío. En los frutos almacenados a $5{ }^{\circ} \mathrm{C}$ esta disminución fue rápida, pasando de un color naranja intenso luego del acondicionado para morado a los 21 días a $5{ }^{\circ} \mathrm{C}$, mientras que los frutos-control cambiaron del naranja para rojizo (Figura 1C y 1D). Esto muestra que esta cultivar es capaz de intensificar el color de la pulpa en postcosecha y que la temperatura influye en la velocidad de producción de pigmentos rojizos, aumentando a $\operatorname{los} 5{ }^{\circ} \mathrm{C}$. En 'Constanza' la formación de color rojiza en la pulpa, sin efecto sobre el sabor, no puede ser relacionada a los daños por frío, estando relacionada a la maduración, como también lo observó Fernández-Trujillo et al. (1998) y LuRie Y CRISOSTO (2005) en duraznos.

Tabla 2. Valores de luminosidad $\left(\mathrm{L}^{*}\right)$, pureza $\left(\mathrm{C}^{*}\right)$ y tonalidad $\left(\mathrm{H}_{\mathrm{ab}}\right)$ para el color de cubrimiento y de la pulpa en ciruelas 'Constanza' durante el almacenaje refrigerado y después de su maduración a $20{ }^{\circ} \mathrm{C}$

\begin{tabular}{|c|c|c|c|c|}
\hline \multirow{2}{*}{ Parámetro } & \multicolumn{2}{|c|}{ Almacenaje } & \multicolumn{2}{|c|}{ Maduración } \\
\hline & Control & Acondicionado & Control & Acondicionado \\
\hline $\mathrm{L}^{*}$ Cubrimiento $0{ }^{\circ} \mathrm{C}$ & $30,9 \mathrm{a}$ & 30,6 a & 30,6 a & 31,9 a \\
\hline $\mathrm{C}^{*}$ Cubrimiento $0{ }^{\circ} \mathrm{C}$ & $6,8 \mathrm{a}$ & $4,8 \mathrm{~b}$ & $5,2 \mathrm{a}$ & $3,8 \mathrm{~b}$ \\
\hline $\mathrm{H}^{*}$ Cubrimiento $0{ }^{\circ} \mathrm{C}$ & $11,8 \mathrm{a}$ & $10,0 \mathrm{a}$ & $10,4 \mathrm{~b}$ & 13,3 a \\
\hline $\mathrm{L}^{*}$ Cubrimiento $5{ }^{\circ} \mathrm{C}$ & $31,6 \mathrm{a}$ & $31,1 \mathrm{a}$ & $30,5 \mathrm{a}$ & $31,1 \mathrm{a}$ \\
\hline $\mathrm{C}^{*}$ Cubrimiento $5^{\circ} \mathrm{C}$ & $6,5 \mathrm{a}$ & $2,8 \mathrm{~b}$ & $4,0 \mathrm{a}$ & $2,8 \mathrm{~b}$ \\
\hline $\mathrm{H}^{*}$ Cubrimiento $5{ }^{\circ} \mathrm{C}$ & $14,6 \mathrm{a}$ & 13,6 a & $14,2 \mathrm{a}$ & $15,4 \mathrm{a}$ \\
\hline $\mathrm{L}^{*}$ pulpa $0{ }^{\circ} \mathrm{C}$ & $48,8 \mathrm{a}$ & $37,8 \mathrm{~b}$ & $41,6 \mathrm{a}$ & $34,9 \mathrm{~b}$ \\
\hline $\mathrm{L}^{*}$ pulpa $5{ }^{\circ} \mathrm{C}$ & $46,1 \mathrm{a}$ & $29,4 \mathrm{~b}$ & $37,5 \mathrm{a}$ & $32,6 \mathrm{~b}$ \\
\hline $\mathrm{C}^{*}$ pulpa $0{ }^{\circ} \mathrm{C}$ & $27,0 \mathrm{a}$ & $22,0 \mathrm{~b}$ & $26,2 \mathrm{a}$ & $22,3 \mathrm{~b}$ \\
\hline $\mathrm{C}^{*}$ pulpa $5^{\circ} \mathrm{C}$ & $28,0 \mathrm{a}$ & $19,2 \mathrm{~b}$ & $24,4 \mathrm{a}$ & $18,8 \mathrm{~b}$ \\
\hline
\end{tabular}

Valores seguidos de la misma letra en la línea, dentro de cada período de evaluación (almacenaje o maduración), no difieren significativamente por el test de Tukey $(p=0,05)$.
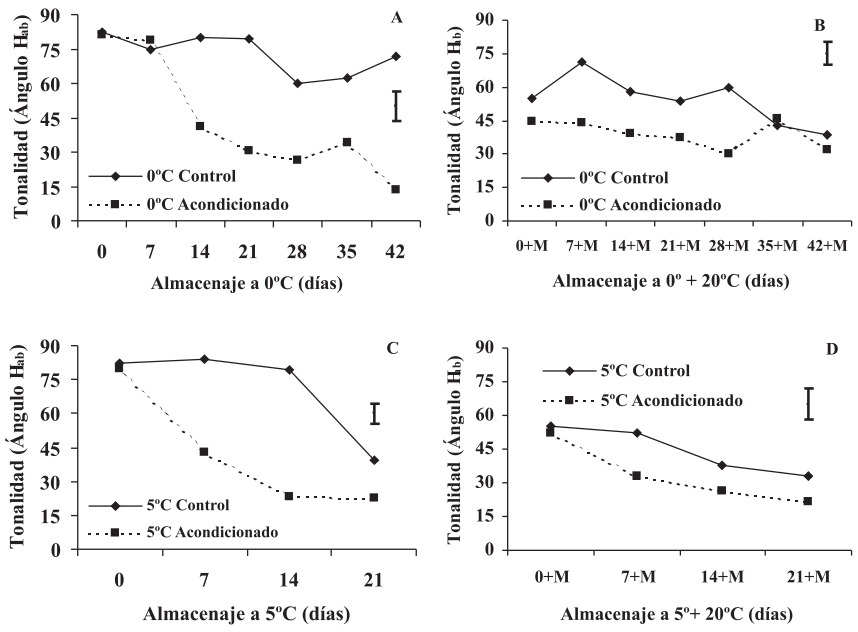

Figura 1. Tonalidad (ángulo $\mathrm{H}_{\mathrm{ab}}$ ) del color de la pulpa en ciruelas 'Constanza' después del almacenaje refrigerado a $0{ }^{\circ} \mathrm{C}(\mathrm{A})$ y $5{ }^{\circ} \mathrm{C}$ (C) y de su respectiva maduración a $20{ }^{\circ} \mathrm{C}(\mathrm{B}$ y $\mathrm{D})$. Barra vertical indica diferencia mínima significativa (Tukey $p=0,05$ ).

'Constanza' presentó frutos con 106,6 gramos de masa promedio y 59,0 $\mathrm{mm}$ de diámetro en el ecuador (Tabla 3). En los sólidos solubles los tratamientos no mostraron diferencias entre si a 0 o 5 ${ }^{\circ} \mathrm{C}$, concordando con las observaciones de RetAMALES et al. (1992) en nectarines sometidos al acondicionado.
Los SST se mantuvieron siempre entre 16 y $17^{\circ}$ Brix sin mayores variaciones. Es posible que la pérdida de azúcares por la respiración haya sido balanceada por el aumento en los azúcares debido a la pérdida de masa, la qual, normalmente, aumenta la concentración de azúcares en los tejidos de la fruta.

En cuanto a la firmeza de la pulpa, las ciruelas del tratamiento control a $0{ }^{\circ} \mathrm{C}$ tuvieron un mayor período de maduración, en promedio de 5 días a $20^{\circ} \mathrm{C}$, respecto a los 3 días de las acondicionadas y dos días de los tratamientos a $5{ }^{\circ} \mathrm{C}$.

La firmeza de la pulpa a la cosecha y durante el almacenaje no presentó grandes diferencias entre los puntos de medición en el fruto. Diferencias significativas ocurrieron sólo entre el hombro y la sutura. Los ecuadores y la región del hombro son los puntos que en promedio presentan la menor firmeza a salida del almacenaje. La sutura y punta (zona distal) del fruto fueron los sectores más firmes. Entre la sutura y los ecuadores hubo una diferencia de 3,1 a 9,1N durante el almacenaje. En los frutos-control hubo diferencia significativa de $17,8 \mathrm{~N}$ entre la sutura y el hombro a partir de los 28 días en frío (Figura 2A). En los frutos acondicionados las diferencias entre el hombro y sutura ocurrieron desde la cosecha (Figura 2B), alcanzando $12,4 \mathrm{~N}$ a los 42 días a $0^{\circ} \mathrm{C}$. 
Tabla 3. Masa, diámetro, sólidos solubles, contenido de jugo promedio en forma visual y harinosidad objetiva en ciruelas 'Constanza' a salida del almacenaje refrigerado a 0 y $5{ }^{\circ} \mathrm{C}(\mathrm{SF})$ y después de maduración a $20^{\circ} \mathrm{C}(\mathrm{M})$

\begin{tabular}{|c|c|c|c|c|c|}
\hline \multirow{2}{*}{ Parámetro } & & \multicolumn{2}{|c|}{ Almacenaje a $0{ }^{\circ} \mathrm{C}$} & \multicolumn{2}{|c|}{ Almacenaje a $5^{\circ} \mathrm{C}$} \\
\hline & & Control & Acondicionado & Control & Acondicionado \\
\hline Masa (g) & SF & 107,2 a & 108,3 a & 103,9 a & 106,8 a \\
\hline Diámetro (mm) & SF & $58,9 \mathrm{a}$ & $59,4 \mathrm{a}$ & $58,4 \mathrm{a}$ & $58,9 \mathrm{a}$ \\
\hline \multirow[t]{2}{*}{ SST ( ${ }^{\circ}$ Brix) } & SF & $16,5 \mathrm{a}$ & $16,1 \mathrm{a}$ & $17,1 \mathrm{a}$ & $16,9 \mathrm{a}$ \\
\hline & $\mathrm{M}$ & $17,0 \mathrm{a}$ & $16,8 \mathrm{a}$ & $16,9 \mathrm{a}$ & $16,7 \mathrm{a}$ \\
\hline \multirow[t]{2}{*}{ Contenido de jugo } & SF & $2,9 \mathrm{a}$ & $2,2 \mathrm{~b}$ & $2,8 \mathrm{a}$ & $1,8 \mathrm{~b}$ \\
\hline & M & $2,7 \mathrm{a}$ & $2,3 \mathrm{~b}$ & $2,2 \mathrm{a}$ & $1,6 \mathrm{~b}$ \\
\hline Harinosidad & $\mathrm{M}$ & $15,9 \mathrm{a}$ & $12,9 \mathrm{a}$ & $12,9 \mathrm{a}$ & $2,1 \mathrm{~b}$ \\
\hline
\end{tabular}

Valores seguidos de la misma letra en la línea, dentro de cada temperatura de almacenaje, no difieren significativamente por el test de Tukey $(p=0,05)$, para cada parámetro.
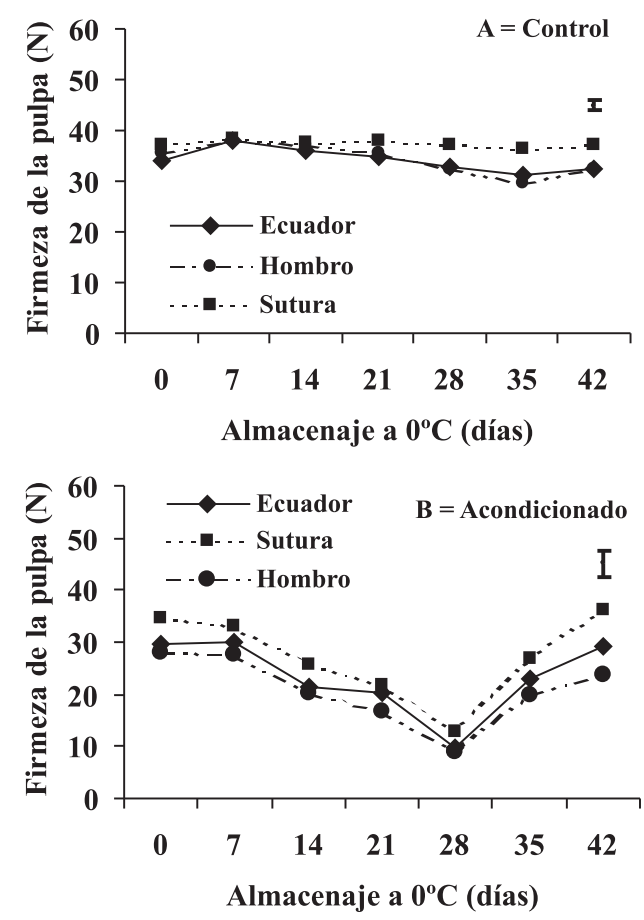

Figura 2. Firmeza de pulpa en la zona del ecuador, hombro y sutura en ciruelas 'Constanza' después del almacenaje refrigerado a $0{ }^{\circ} \mathrm{C}$. Barra vertical indica diferencia mínima significativa (Tukey $p=0,05$ ).

En los ecuadores del fruto, las ciruelas-control presentaron firmeza superior a las acondicionadas a salida del almacenaje refrigerado a $0{ }^{\circ} \mathrm{C}$ (Figura 3A), comportamiento que se mantuvo en parte durante la maduración (Figura 3B). Los frutos-control cambiaron poco la firmeza en la conservación, manteniendo los valores entre 38 y $31 \mathrm{~N}$. En las ciruelas acondicionadas la firmeza disminuyó del final del calentamiento hasta los 28 días a $0^{\circ} \mathrm{C}$ cuando alcanzó $9 \mathrm{~N}$, pero retornó a los niveles de la cosecha a los 35 y 42 días en frío. Esta firmeza a los 28 días, considerada baja para comercialización, ocurrió debido a una mayor maduración de algunas ciruelas en esta fecha de evaluación.
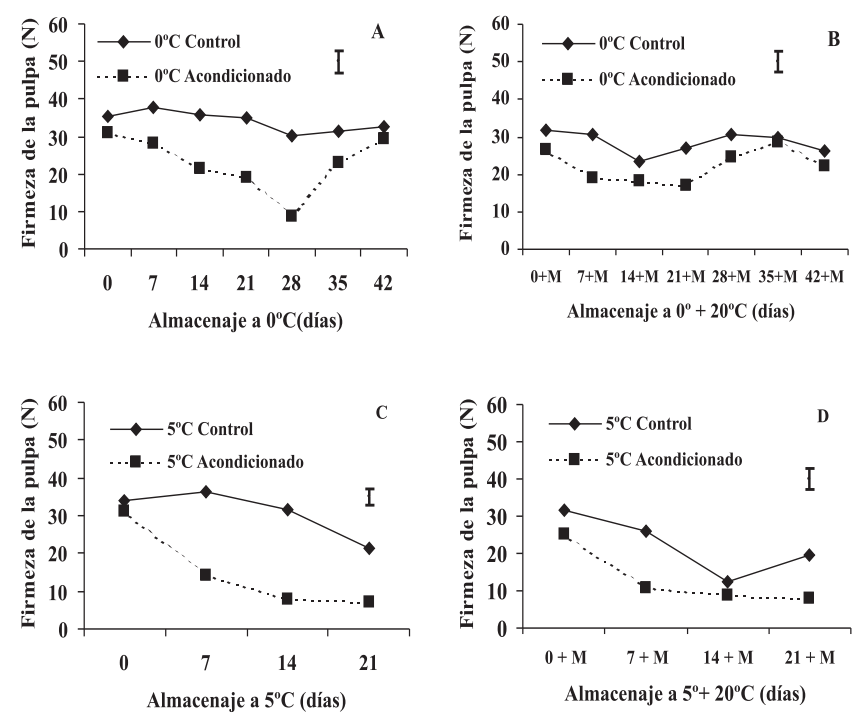

Figura 3. Firmeza de la pulpa en el sector de los ecuadores del fruto en ciruelas 'Constanza' después del almacenaje refrigerado a 0 y $5{ }^{\circ} \mathrm{C}$ (A y C) y de su respectiva maduración a $20{ }^{\circ} \mathrm{C}$ (B y D). Barra vertical indica diferencia mínima significativa (Tukey $p=0,05$ ).

La firmeza en la zona de los ecuadores de las ciruelas almacenadas a $5{ }^{\circ} \mathrm{C}$ disminuyó en los 21 días de conservación, siendo más intensa en los frutos acondicionados, que presentaron una pérdida de $24 \mathrm{~N}$ al largo del almacenaje, contra $12 \mathrm{~N}$ de los controles (Figura 3C). Este comportamiento fue semejante en la maduración, pero al disminuir la firmeza fue más intenso en los frutos-control (Figura 3D). Las ciruelas acondicionadas en la conservación a partir de los 14 días a $5{ }^{\circ} \mathrm{C}$ y en toda la maduración después de 7 días a $5{ }^{\circ} \mathrm{C}$ presentaron un $50 \%$ de frutos sobre maduros con 
firmeza inferior a $9,0 \mathrm{~N}$, considerada buena para consumo, pero baja para comercialización. La elevada pérdida de firmeza es un factor que dificulta el almacenaje prolongado. Retamales et al. (1992) reportaron gran pérdida de firmeza en nectarines calentados antes del almacenaje refrigerado. A pesar de haber disminuido poco en los seis días de calentamiento, la combinación con el almacenaje a $5{ }^{\circ} \mathrm{C}$ permitió que la firmeza disminuya más rápidamente, tanto en la conservación como en la maduración, comparado al almacenaje a $0{ }^{\circ} \mathrm{C}$, indicando que el acondicionado lleva a diferentes grados de ablandamiento dependiendo de la temperatura de almacenaje.

La intensidad de jugosidad evaluada visualmente fue superior en los frutos calentados. La jugosidad fue baja en las ciruelas-control y moderada en las acondicionadas a $0{ }^{\circ} \mathrm{C}$ y en su maduración (Tabla 3). En los frutos almacenados a $5{ }^{\circ} \mathrm{C}$ fue moderada a baja a la salida de frío en los frutoscontrol y moderada para los acondicionados, pero en la maduración fue moderada en los frutos-control y alta para los acondicionados.

El contenido de jugo extraído por centrifugación en los frutos almacenados a $0{ }^{\circ} \mathrm{C}$ fue significativamente superior en los frutos acondicionados a los 7,14 y 21 días a $0{ }^{\circ} \mathrm{C}$ comparado a los controles, igualando el contenido de jugo a partir de los 28 días de almacenaje (Figura 4A). Durante el almacenaje, el jugo extraído de los frutos acondicionados fue de $46 \%$, significativamente superior a los $41 \%$ de los controles. En la maduración, los tratamientos no mostraron diferencias entre si (Figura 4B), pero los frutos calentados presentaron $9,0 \%$ más jugo en los primeros 21 días del ensayo. En los frutos almacenados a $5{ }^{\circ} \mathrm{C}$ no ocurrieron diferencias de jugo extraído durante el almacenaje y la maduración (Figuras 4C y 4D), siendo el contenido de jugo siempre superior a los $38 \%$. Los frutos acondicionados presentaron promedio de $5 \%$ más de jugo que los controles en la maduración. El acondicionado normalmente aumenta el contenido de jugo de los frutos como observaron Von MollendorfF et al. (1992), Ju et al. (2000), ZHou et al. $(1999,2000)$ y Crisosto el al. (2004), pero en 'Constanza', principalmente en las ciruelas almacenadas a $5{ }^{\circ} \mathrm{C}$, este tratamiento no presentó efecto en aumentar la cantidad de jugo.

Adicionalmente, junto con la medición de jugosidad, se observó que la viscosidad del jugo extraído fue alta en algunas ciruelas, con difícil escurrimiento cuando se pesó para su cuantificación. Este aspecto empezó a ser visualizado en los días de maduración siguientes a la cosecha en los frutos acondicionados (datos no presentados). El mayor porcentaje de ciruelas con jugo viscoso fue observado en la maduración pasados 14 días a $5{ }^{\circ} \mathrm{C}$, alcanzando $50 \%$ de los frutos acondicionados. Algunos investigadores como, LuCHSINGER y WALSH (1997), ZHOU et al. (1999) afirman que el jugo viscoso es una característica de frutas con harinosidad. Von Mollendorff y De Villiers (1988) observaron que la viscosidad de las pectinas solubles alcanzó valores máximos durante el desarrollo de harinosidad y que en frutos sin el daño el aumento en la viscosidad fue menor.
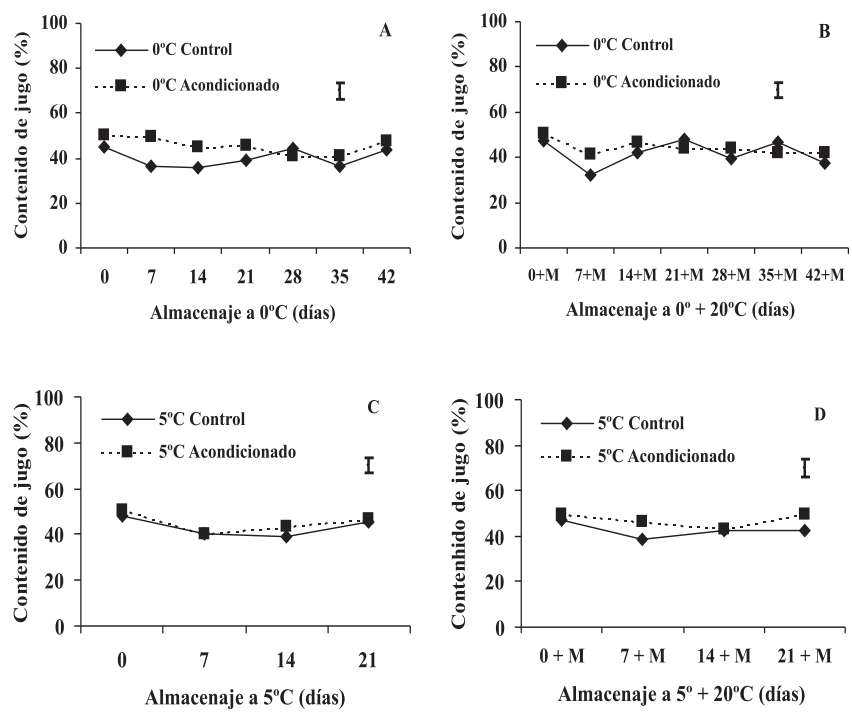

Figura 4. Contenido de jugo (\%) en ciruelas 'Constanza' después del almacenaje refrigerado a 0 y $5{ }^{\circ} \mathrm{C}$ (A y C) y de su respectiva maduración a $20{ }^{\circ} \mathrm{C}$ (B y D). Barra vertical indica diferencia mínima significativa (Tukey $p=0,05)$.

Frutos del cv. Constanza fueron susceptibles a los daños por frío, siendo el pardeamiento, la harinosidad y la retención de firmeza los desórdenes fisiológicos determinados en las ciruelas a partir de 14 días de almacenaje. Externamente los frutos con daño no difirieron de los normales.

Pardeamiento de la pulpa fue observado en $50 \%$ de los frutos-control, que presentaron la pulpa parda alrededor del carozo, maduración a los 42 días a $0{ }^{\circ} \mathrm{C}$ más 5 días a $20^{\circ} \mathrm{C}$, de intensidad moderada, en el tratamiento. Pardeamiento interno es un daño común en frutas de carozo, pero CRISOsto el al. (1999) señalan que frutos de ninguna cultivar de ciruelas que estudiaron desarrollo este daño al ser almacenadas a $0{ }^{\circ} \mathrm{C}$ por 4 semanas. En ciruelas 'Santa Rosa' almacenadas a $0{ }^{\circ} \mathrm{C}$ continuamente o a 0 y $3{ }^{\circ} \mathrm{C}$ en calentamiento intermitente los daños por frío se caracterizaron como pardeamiento del mesocarpo (KLUGE et al., 1997). 
El pardeamiento consiste en la oxidación de substratos fenólicos a $o$-quinonas que se condensan y combinan con grupos amino o sulfidrilo de las proteínas y con azúcares reductores formando polímeros pardos. El proceso se desencadena cuando se ponen en contacto los substratos fenólicos con las enzimas que catalizan las reacciones de oxidación de los polifenoles (ArTÉs et al., 1998). Como el pardeamiento en 'Constanza' se manifestó en la última evaluación, este puede haber suido resultado del avanzado tiempo en conservación a que la fruta fue sometida e indicaría que alcanzó su período máximo de conservación y las estructuras celulares empezaron a colapsar (Tabla 4).

Tabla 4. Porcentaje de ciruelas 'Constanza' sanas, con pardeamiento interno, retención de firmeza y harinosidad en la maduración a $20{ }^{\circ} \mathrm{C}(\mathrm{M})$ de frutos almacenados a $0{ }^{\circ} \mathrm{C}$ y $5{ }^{\circ} \mathrm{C}$

\begin{tabular}{|c|c|c|c|c|c|c|c|c|}
\hline \multirow{3}{*}{$\begin{array}{l}\text { Fechas de } \\
\text { evaluación }\end{array}$} & \multicolumn{8}{|c|}{ Almacenaje a $0{ }^{\circ} \mathrm{C}$} \\
\hline & \multicolumn{2}{|c|}{ Pardeamiento } & \multicolumn{2}{|c|}{ Harinosidad } & \multicolumn{2}{|c|}{ Retención } & \multicolumn{2}{|c|}{ Sanas } \\
\hline & $\mathrm{C}$ & $\mathrm{AC}$ & $\mathrm{C}$ & $\mathrm{AC}$ & $\mathrm{C}$ & $\mathrm{AC}$ & $\mathrm{C}$ & $\mathrm{AC}$ \\
\hline $7+M$ & $0 \mathrm{a}$ & $0 \mathrm{a}$ & $0 \mathrm{a}$ & $0 \mathrm{a}$ & $0 \mathrm{a}$ & $0 \mathrm{a}$ & $100 \mathrm{a}$ & $100 \mathrm{a}$ \\
\hline $14+\mathrm{M}$ & $0 \mathrm{a}$ & $0 \mathrm{a}$ & $20 \mathrm{a}$ & $10 \mathrm{a}$ & $0 \mathrm{a}$ & $0 \mathrm{a}$ & $80 \mathrm{a}$ & $90 \mathrm{a}$ \\
\hline $21+M$ & $0 \mathrm{a}$ & $0 \mathrm{a}$ & $0 \mathrm{~b}$ & $30 \mathrm{a}$ & $0 \mathrm{a}$ & $0 \mathrm{a}$ & $100 \mathrm{a}$ & $70 \mathrm{~b}$ \\
\hline $28+M$ & $0 \mathrm{a}$ & $0 \mathrm{a}$ & $0 \mathrm{a}$ & $0 \mathrm{a}$ & $30 \mathrm{a}$ & $0 \mathrm{~b}$ & $70 \mathrm{~b}$ & $100 \mathrm{a}$ \\
\hline $35+M$ & $0 \mathrm{a}$ & $0 \mathrm{a}$ & $0 \mathrm{a}$ & $0 \mathrm{a}$ & $20 \mathrm{a}$ & $0 \mathrm{~b}$ & $80 \mathrm{a}$ & $100 \mathrm{a}$ \\
\hline $42+M$ & $50 \mathrm{a}$ & $0 \mathrm{~b}$ & $0 \mathrm{a}$ & $10 \mathrm{a}$ & $20 \mathrm{a}$ & $20 \mathrm{a}$ & $50 \mathrm{a}$ & $70 \mathrm{a}$ \\
\hline Promedio & $8,3 \mathrm{a}$ & $0 \mathrm{~b}$ & $3,3 \mathrm{a}$ & $8,3 \mathrm{a}$ & $11,7 \mathrm{a}$ & $2,3 \mathrm{~b}$ & $80 \mathrm{a}$ & 88,3 a \\
\hline \multirow{3}{*}{$\begin{array}{l}\text { Fechas de } \\
\text { evaluación }\end{array}$} & \multicolumn{8}{|c|}{ Almacenaje a $5^{\circ} \mathrm{C}$} \\
\hline & \multicolumn{2}{|c|}{ Pardeamiento } & \multicolumn{2}{|c|}{ Harinosidad } & \multicolumn{2}{|c|}{ Retención } & \multicolumn{2}{|c|}{ Sanas } \\
\hline & $\mathrm{C}$ & $\mathrm{AC}$ & $\mathrm{C}$ & $\mathrm{AC}$ & $\mathrm{C}$ & $\mathrm{AC}$ & $\mathrm{C}$ & $\mathrm{AC}$ \\
\hline $7+M$ & 0 & 0 & $0 \mathrm{a}$ & $0 \mathrm{a}$ & 0 & 0 & $100 \mathrm{a}$ & $100 \mathrm{a}$ \\
\hline $14+\mathrm{M}$ & 0 & 0 & $20 \mathrm{a}$ & $50 \mathrm{a}$ & 0 & 0 & $80 \mathrm{a}$ & $50 \mathrm{a}$ \\
\hline $21+M$ & 0 & 0 & $10 \mathrm{a}$ & $20 \mathrm{a}$ & 0 & 0 & $100 \mathrm{a}$ & $80 \mathrm{a}$ \\
\hline Promedio & 0 & 0 & $10 \mathrm{a}$ & $23,3 \mathrm{a}$ & 0 & 0 & $90 \mathrm{a}$ & $76,7 \mathrm{a}$ \\
\hline
\end{tabular}

Valores seguidos de la misma letra en la línea, no difieren significativamente por el test de Tukey $(p=0,05)$. C = Control; AC = Acondicionado; $\mathrm{M}=5$ días para frutos controles almacenados a $0{ }^{\circ} \mathrm{C}, 3$ días para frutos acondicionados almacenados a $0{ }^{\circ} \mathrm{C}, 2$ días para frutos-control almacenados a $5{ }^{\circ} \mathrm{C}$ y 2 días para frutos acondicionados almacenados a $5{ }^{\circ} \mathrm{C}$.

'Constanza' presentó harinosidad, con pulpa seca y harinosa, en todos los tratamientos en la maduración a $20{ }^{\circ} \mathrm{C}$ pasados los 14 días de conservación (Tabla 4). En todas las frutas el grado de harinosidad fue de 3 , con daño moderado. Las ciruelas con harinosidad tenían su jugo viscoso. A salida de frío los frutos almacenados a $5{ }^{\circ} \mathrm{C}$ y acondicionados, después de 14 y 21 días de conservación, presentaron $10 \%$ y $20 \%$ de fruta harinosa, respectivamente (datos no presentados). Normalmente los síntomas de harinosidad no son visualizados durante o luego del almacenaje refrigerado, sino más bien después de 1 a 2 días de maduración a $20^{\circ} \mathrm{C}$ (LUCHSINGER y WALSH, 1997). Según CRisosto et al. (2004), son de gran importancia para el aparecimiento de daños por frío el estado de madurez, el tiempo y temperatura de almacenaje y la susceptibilidad de cada variedad. Así, considerando el estado avanzado de maduración de los frutos almacenados a $5{ }^{\circ} \mathrm{C}$, es posible aceptar la manifestación de harinosidad a la salida del almacenaje en frío. RETAMALEs et al. (1992) observaron harinosidad en nectarines a salida de frío y lo explicaron debido a la susceptibilidad de las variedades.

Harinosidad por análisis visual se constituyó un problema en un mayor porcentaje de frutos por fecha de evaluación en las ciruelas acondicionadas, pero sin diferencias para los controles (Tabla 4). Por el análisis objetivo con base en el contenido de jugo no se presentaron diferencias entre los frutos a $0{ }^{\circ} \mathrm{C}$, en cuanto en las ciruelas acondicionadas almacenadas a $5{ }^{\circ} \mathrm{C}$ la intensidad de la harinosidad fue menor que en los frutos del control (Tabla 3). En los trabajos de Streif et al. (1994), Retamales et al. (1992) y CRIsosto el al. (2004) se observó que el acondicionado de frutos previo el almacenaje en frío 
disminuye la incidencia de daños por frío. CRISOSTO el al. (1999) analizando la susceptibilidad de frutas de carozo a los daños por frío no observaron harinosidad en cultivares de ciruelas almacenadas a $0{ }^{\circ} \mathrm{C}$ por 4 semanas. Eso indica que se deben realizar los estudios en forma específica para cada especie y variedad a modo de evitar generalizaciones.

Se observaron valores elevados en la firmeza, considerados anormales, en frutos-control en la maduración a $20^{\circ} \mathrm{C}$, después de 28,35 y 42 días a 0 ${ }^{\circ} \mathrm{C}$, en el 30,20 y $20 \%$ de los frutos, respectivamente. Además, en el $20 \%$ de los frutos acondicionados después de 42 días a $0{ }^{\circ} \mathrm{C}$ más 3 días de maduración (Tabla 4). La pulpa de estos frutos presentaba un aspecto tipo corcho o cuerudo (leatheriness). Esto puede haber sido otro síntoma de desorden causado por el frío que es la retención de firmeza, que afectó más a los frutos-control. Según LuchSINGER y WALSH (1997), a $0{ }^{\circ} \mathrm{C}$ se produce una fuerte inhibición de la maduración, producto de la baja tasa de producción de etileno con una retención de la firmeza del fruto lo cual provoca un retraso en la expresión del síntoma de harinosidad. Según Ju et al. (2000) harinosidad y retención de firmeza son diferentes daños causados por el frío y, muchas veces, puede haber confusión en distinguir los síntomas de cada uno. Según Ju et al. (2000), la retención de firmeza ocurre cuando los frutos mantienen una firmeza superior a $40 \mathrm{~N}$. En ciruelas 'Constanza' que presentaron el daño, este síntoma, pulpa con aspecto de corcho, se observó en frutos con firmeza superior a 36N (Figura 3B), valores anormalmente elevados debido al daño. Anomalías semejantes en la firmeza, fueron descritas también por Fernandez-Trujillo et al. (1998) en duraznos que sufrieron daños por frío.

En la literatura el acondicionado previne o atrasa la manifestación de daños por frío y, así, aumenta el porcentaje de frutos sanos comercializables (Lurie y Crisosto, 2005). Pero en 'Constanza' no ocurrieron diferencias en el porcentaje de frutos sanos entre los tratamientos, sea a 0 o $5^{\circ} \mathrm{C}$. Sin embargo, el acondicionado combinado con el almacenaje a $0{ }^{\circ} \mathrm{C}$ proporcionó un aumento en el porcentaje de frutos sanos en las fechas finales de evaluación, respecto al control, pues hubo un aumento de $20 \%$ a $30 \%$ en el porcentaje de frutos sanos en las fechas de mayor manifestación de los daños.

Las ciruelas acondicionadas y almacenadas a $5{ }^{\circ} \mathrm{C}$ tuvieron aún algunos frutos muy maduros, con firmeza igual o inferior a $9 \mathrm{~N}$ en el $30 \%$; $70 \%$ y $70 \%$ de frutos a los 7, 14 y 21 días en frío, respectivamente, y en la maduración de estas mismas fechas en el $50 \%$; $60 \%$ y $70 \%$ respectivamente. En los controles almacenados a $5{ }^{\circ} \mathrm{C}$ hubo $20 \%$ de frutos sobremaduros a los 21 días de almacenaje más 2 días a $20^{\circ} \mathrm{C}$. En los frutos acondicionados almacenados a $0{ }^{\circ} \mathrm{C}$ hubo $50 \%$ de ciruelas con baja firmeza a los 28 días de frío. Debido a la baja firmeza, estos frutos no son comercializables o necesitan ser comercializados en embalajes especiales, a modo de evitar daños por manipulación.

\section{CONCLUSIONES}

1. El acondicionado previo el almacenaje no aumenta la pérdida de masa en ciruelas 'Constanza' durante el almacenaje refrigerado a 0 o $5^{\circ} \mathrm{C}$.

2. El acondicionado no afecta la calidad de los frutos almacenados a $0{ }^{\circ} \mathrm{C}$, pero cuando se almacenan a $5^{\circ} \mathrm{C}$ causa una gran pérdida de firmeza en ciruelas 'Constanza'.

3. El acondicionado proporciona un aumento en el porcentaje de frutos sanos comercializables en los períodos finales de maduración de ciruelas 'Constanza' almacenadas a $0{ }^{\circ} \mathrm{C}$.

\section{REFERENCIAS}

ARTÉS, F.; CASTAÑER, M.; GIL, M.I. Revisión: El pardeamiento enzimático en frutas y hortalizas mínimamente procesadas. Food Science and Technology International, London, v.4, n.6, p.377-389. 1998.

BRUHN, C.M.; FELDMAN, N.; GARLITZ, C.; HARDWOOD, J.; IVAN, E.; MARSHALL, M; RILEY, A.; THURBER, D.; WILLIAMSON, E. Consumer perceptions of quality: Apricots, cantaloupes, peaches, pears, strawberries and tomatoes. Journal of Food Quality, Trumbull, v.14, n.2, p.187-195, 1991.

CRISOSTO, C.H.; MITCHELL, F.G.; JU, Z. Susceptibility to chilling injury of peach, nectarine, and plum cultivars grown in California. HortScience, Alexandria, v.34, n.6, p.1116-1118, 1999.

CRISOSTO, C.H.; GARNER, D.; ANDRIS, H.L.; DAY, K.R. Controlled delayed cooling extends peach market life. HortTechnology, Alexandria, v.14, n.1, p.99-104, 2004.

FAO, 2006. FAOSTAT Agriculture. Agricultural Data Agricultural Production. In: http://faostat.fao.org/faostat/ collections?subset=agriculture. Accesado en 20/04/2006.

FERNÁNDEZ-TRUJILLO, J.P.; MARTÍNEZ, J.A.; ARTÉS, F. Efectos de la conservación frigorífica en la fisiología y calidad del melocotón Sudanell. Food Science and Technology International, Frederick, v.4, n.4, p.245-255, 1998.

JU, Z.; DUAN, Y.; JU, Z. Leatheriness and mealiness of peaches in relation to fruit maturity and storage temperature. Journal of Horticultural Science \& Biotechnology, Ashford, v.75, n.1, p.86-91, 2000. 
KLUGE, R.A.; CANTILLANO, R.F.F.; BILHALVA, A.B. Colapso de polpa em ameixas 'Santa Rosa' armazenadas em diferentes regimes de temperatura. Revista Brasileira de Agrociência, v.3, n.3, Pelotas, p.125-130, 1997.

LILL, R.E.; VAN DER MESPEL, G.J. A method for measuring the juice content of mealy nectarines. Scientia Horticulturae, Amsterdam, v.36, n.3-4, p.267-271, 1988.

LIZANA, L. A.; RODRIGUEZ, R. Efecto de la temperatura en la predisposición al desarrollo de desórdenes fisiológicos en ciruelas japonesas cv. Casselman de exportación. In: SIMPOSIO INTERNACIONAL DE MANEJO, CALIDAD Y FISIOLOGÍA POSTCOSECHA DE FRUTAS, 4., 1995. Publicaciones Misceláneas Agrícolas, Universidad de Chile, Santiago, n.42, p.45-54, 1995.

LUCHSINGER, L. Determinación objetiva de la harinosidad en frutos de carozo mediante la relación entre el contenido de jugo y firmeza del fruto. Simiente, Santiago, v.70, n.3-4, p.127$128,2000$.

LUCHSINGER, L; WALSH, C.S. Problemática de la exportación de duraznos, nectarines y ciruelas. II Parte: Desórdenes fisiológicos. Aconex, Santiago, v.56, n.3, p.27-32, 1997.

LURIE, S.; CRISOSTO, C.H. Chilling injury in peach and nectarine. Postharvest Biology and Technology, Amsterdam, v.37, n.3, p.195-208, 2005

RETAMALES, J.; COOPER, T.; STREIF, J.; KANIA, J.C. Preventing cold storage disorders in nectarines. Journal of Horticultural Science, Ashford, v.67, n.5, p.619-626, 1992.
STREIF, J.; RETAMALES, J.; COOPER, T. Preventing cold storage disorders in nectarines. Acta Horticulturae, Leuven, n.368, p.160-166, 1994.

VON MOLLENDORF, L.J.; DE VILLIERS, O.T. Role of pectolytic enzymes in the development of wooliness in peaches. Journal of Horticultural Science, Ashford, n.63, p.53-58, 1988.

VON MOLLENDORFF, L.J.; JACOBS, G.; DE VILLIERS, O.T. Effect of temperature manipulation during storage and ripening on firmness, extractable juice and woolliness in nectarines. Journal of Horticultural Science, Ashford, v.67, n.5, p.655-662, 1992.

ZHOU, H-W.; SONEGO, L.; BEN-ARIE, R.; LURIE, S. Analysis of cell wall components in juice of 'Flavortop' nectarines during normal ripening and woolliness development. Journal of the American Society for Horticultural Science, Alexandria, v.124, n.4, p.424-429, 1999.

ZHOU, H-W.; LURIE, S.; LERS, A; KHATCHITSKI, A; SONEGO, L.; BEN-ARIE, R. Delayed storage and controlled atmosphere storage of nectarines: two strategies to prevent woolliness. Postharvest Biology and Technology, Amsterdam, v.18, n.2, p.133-141, 2000.

ZONTA, E.P.; MACHADO, A.A. Sistema de Análise Estatística para microcomputadores - "SANEST" (software). Pelotas: UFPEL, Instituto de Física e Matemática, 1986. 